\title{
Iron overload impairs normal hematopoietic stem and progenitor cells through reactive oxygen species and shortens survival in myelodysplastic syndrome mice
}

\author{
Xin Jin, ${ }^{1+}$ Xiaoyuan He,${ }^{1+}$ Xiaoli Cao, ${ }^{2}$ Ping Xu, ${ }^{3}$ Yi Xing, ${ }^{2}$ Songnan Sui, ${ }^{3}$ \\ Luqiao Wang, ${ }^{3}$ Juanxia Meng, ${ }^{3}$ Wenyi Lu, ${ }^{3}$ Rui Cui, ${ }^{3}$ Hongyan $\mathrm{Ni}^{4 * \star}$ \\ and Mingfeng Zhao $\mathrm{Zn}^{3,1^{* *}}$
} ${ }^{1}$ Nankai University School of Medicine, Tianjin; ${ }^{2}$ Tianjin Children's Hospital; ${ }^{3}$ Department
of Hematology, Tianjin First Central Hospital and ${ }^{4}$ Department of Radiology, Tianjin First
Central Hospital, Tianjin, PR China

$* \mathrm{XJ}$ and $\mathrm{XYH}$, and **HYN and MFZ contributed equally to this work.

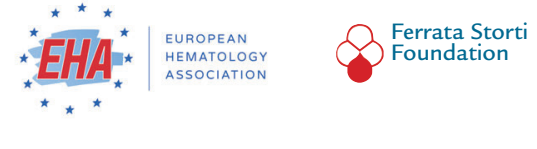

Haematologica 2018

Volume 103(10):1627-1634

\section{ABSTRACT}

There is increasing clinical evidence to suggest a suppressive effect on hematopoiesis in myelodysplastic syndrome patients with iron overload. However, how iron overload influences hematopoiesis in myelodysplastic syndrome (MDS) remains unknown. Here, the RUNX1S291fs-transduced bone marrow mononuclear cells were yielded and transplanted into lethally irradiated recipient mice together with radioprotective bone marrow cells to generate MDS mice. Eight weeks post transplantation, the recipient mice received an intraperitoneal injection of $0.2 \mathrm{~mL}$ iron dextran at a concentration of $25 \mathrm{mg} / \mathrm{mL}$ once every other day for a total of 8 times to establish an iron overload model. In the present study, we show that iron overload impairs the frequency and colony-forming capacity of normal hematopoietic stem and progenitor cells, especially in erythroid, in MDS mice, which is due, at least in part, to growth differentiation factor 11 -induced reactive oxygen species, shortening survival of MDS mice. Given that we are the first to construct an iron overload model in MDS mice, we hope this model will be helpful for further exploring the influence and mechanism of iron overload on MDS.

\section{Introduction}

Myelodysplastic syndrome (MDS) is a heterogeneous group of myeloid malignancy characterized by dysplastic changes in one or more cell lineages, ineffective hematopoiesis, and high risk of leukemic transformation. ${ }^{1,2}$ Because of chronic anemia, up to $80 \%$ of MDS patients become transfusion dependent. ${ }^{3}$ Due to repeated blood transfusion and ineffective hematopoiesis, most MDS patients can eventually develop iron overload. Excessive iron can deposit in the liver, heart, spleen, pancreas, bone marrow (BM) and other tissues, resulting in tissue damage and fibrosis, and a series of complications which seriously affect the prognosis of MDS patients. ${ }^{4-6}$ Therefore, iron overload in MDS patients has received great attention from clinicians. Here the question is whether and how iron overload impacts the hematopoietic system in MDS. Indeed, iron-chelation therapy often improves the prognosis of MDS patients, implicating that iron overload has a suppressive effect on hematopoiesis in these subjects. However, how iron overload influences hematopoiesis in MDS still has to be addressed.

RUNX1 (also known as AML1/CBFA2), one of the most common mutation genes in MDS patients, has an important role in hematopoiesis. RUNX1-S291fs is one type of RUNX1 mutation. Previous studies have proved that RUNX1-S291fs mutant can be used to construct an MDS mouse model. ${ }^{7,8}$ In order to investigate how iron overload affects hematopoiesis in MDS, we generated a RUNX1-S291fsinduced MDS mouse model and subsequently administered iron dextran by intraperitoneal injection to establish an iron overload model in MDS mice.

In this study, we found that iron overload impairs the frequency and function of

\section{Correspondence:}

mingfengzhao@sina.com

Received: March 10, 2018.

Accepted: June 7, 2018.

Pre-published: June 14, 2018.

doi:10.3324/haematol.2018.193128

Check the online version for the most updated information on this article, online supplements, and information on authorship \& disclosures: www.haematologica.org/content/103/10/1627

\section{(C)2018 Ferrata Storti Foundation}

Material published in Haematologica is covered by copyright. All rights are reserved to the Ferrata Storti Foundation. Use of published material is allowed under the following terms and conditions:

https://creativecommons.org/licenses/by-nc/4.0/legalcode. Copies of published material are allowed for personal or internal use. Sharing published material for non-commercial purposes is subject to the following conditions:

https://creativecommons.org/licenses/by-nc/4.0/legalcode, sect. 3. Reproducing and sharing published material for commercial purposes is not allowed without permission in writing from the publisher. 
normal hematopoietic stem and progenitor cells (HSPCs), especially in erythroid cells, which may result from growth differentiation factor 11(GDF11)-induced reactive oxygen species (ROS) and shortens survival in MDS mice. Here we are the first to utilize RUNX1-S291fs-induced MDS mice to successfully construct an iron overload model. This can provide the experimental basis for further exploring the influence and mechanism of iron overload on MDS.

\section{Methods}

\section{Ethical considerations}

Our experiment has been approved by the Ethics Committee of Tianjin First Central Hospital. All animal experiments were conducted in accordance with a protocol approved by the Institutional Animal Care and Use Committee (IACUC) of the Institute of Radiation Medicine, Chinese Academy of Medical Sciences (n. 1204).

\section{Vector and retrovirus construct}

The Empty control vector ( $p M Y s$-Empty control-IRES-GFP) and the RUNX1-S291fs mutant vector (pMYs-RUNX1 S291fs-IRESGFP) were kindly provided by Dr Atsushi Iwam (Kumamoto University, Japan). Production of retrovirus construct has been described previously. ${ }^{8}$

\section{Mice and treatment}

C57BL/6 mice were purchased from Vital River (Beijing, China) at 6-8 weeks of age. The animals were quarantined and allowed to acclimatize for one week. They were maintained in a room at $22 \pm 2^{\circ} \mathrm{C}$, with a relative humidity of $50 \% \pm 10 \%$. The mice were housed 5 individuals per cage and used at a weight of approximately 20.0-22.0g. Retroviral construct transduced into bone marrow mononuclear cells (BMMNCs) was performed as previously described. ${ }^{9}$ Briefly, retrovirus-infected BMMNCs were cultured in aMEM medium containing $10 \mathrm{ng} / \mathrm{mL}$ of human IL-6, $10 \mathrm{ng} / \mathrm{mL}$ of mouse IL-3, and $100 \mathrm{ng} / \mathrm{mL}$ of mouse SCF for three days, and then these cells were intravenously injected into 9Gy lethally irradiated recipient mice together with radioprotective dose of $1 \times 106 \mathrm{BM}$ cells to generate MDS mice or control mice. Eight weeks post transplantation, the recipient mice were administered an intraperitoneal injection of $0.2 \mathrm{~mL}$ iron dextran at a concentration of 25 $\mathrm{mg} / \mathrm{mL}$ once every other day for a total of 8 times to establish an iron overload model. At the same time, normal saline was given to the control group.

\section{Flow cytometric analysis}

Flow cytometry was performed by using the following monoclonal mouse antibodies: CD45.2, CD45.1, Gr1, Mac1/CD11b, Ter119, CD71, B220, CD19, CD3, CD5, CD41, CD117/c-Kit, Sca1 and CD34. All Flow cytometric analysis of the stained cells was performed with Coulter Altra (Beckman Coulter) equipped with Cytexpert software (Beckman Coulter).

\section{Western blot analysis}

To detect the expression of RUNX1S291fs protein, BM cells and spleen cells were lysed and blotted as previously described. ${ }^{10}$ Polyclonal anti-RUNX1 and anti- $\alpha$-Tubulin antibodies were used to detect RUNX1S291fs protein and $\alpha$-Tubulin, respectively.

\section{Diagnosis}

The diagnosis was made according to the Bethesda proposals for classification of MDS in mice. ${ }^{11}$

\section{Statistical analysis}

Results were analyzed with the GraphPad Prism program (GraphPad Software, Inc., San Diego, CA, USA). Data obeyed normal distribution were presented as means \pm Standard Deviation (SD) and multiple group comparisons were performed by using one-way analysis of variance (ANOVA), whereas data with nonnormal distribution were showed by median and quartiles, and compared by Kruskal-Wallis test. The survival curves were analyzed using the Kaplan-Meier method with the log-rank test. $P<0.05$ was considered statistically significant.

\section{Results}

\section{Iron overload model can be established in RUNX1S291fs-induced MDS mice}

In order to explore the role of iron overload in MDS, we first transduced a $p M Y s-R U N X 1 S 291 f_{s}$-IRES-GFP or a pMYs-Empty control-IRES-GFP retroviral construct into BMMNCs (Figure 1A). After five days culture in vitro, the transduced cells were transplanted into lethally irradiated recipient mice together with radioprotective $\mathrm{BM}$ cells to generate $\mathrm{MDS}$ mice or control mice (Figure 1A). Eight weeks after transplantation, the recipient mice were administered iron dextran or normal saline by intraperitoneal injection. Hereafter, we refer to the Empty control mice with normal saline, the Empty control mice with iron dextran, the RUNX1S291fs mice with normal saline, and the RUNX1S291fs mice with iron dextran as Empty/NS, Empty/FE, RX291/NS and RX291/FE mice, respectively. The ratio of $\mathrm{GFP}^{+}$cells gradually increased over 24 weeks after the transplantation in RX291/NS and RX291/FE mice, but not in mice who received transplants of the Empty control-transduced cells (Figure 1B), which indicated that the RUNX1S291fs-transduced cells successfully engrafted and established chimerism in the peripheral blood (PB).

To verify the establishment of an MDS model, several mice were sacrificed at 24 weeks post transplantation. The RUNX1S291fs protein was detected high expression in spleen and low expression in BM in RX291/NS and RX291/FE groups, but not in Empty/NS and Empty/FE mice (Figure 1C), suggesting that RUNX1S291fs was successfully transduced and expressed. We then examined whether RUNX1S291fs contributes to the development of $M D S$ in recipient mice. The mice received RUNX1S291fstransduced cells showed significantly reduced white blood cell (WBC) counts (Figure 1D), hemoglobin (HG) levels (Figure 1E), platelet counts (PLT) (Figure 1F), and increased mean corpuscular volume (MCV) (Figure 1G) compared with Empty control recipient mice. The RUNX1S291fs mutant mice also showed dysplastic cells, such as dot-like and polychromatic erythrocytes, giant platelets, pseudoPelger-Huet granulocytes in the PB, together with dualnucleated granulocytes, increased immature blasts (less than $20 \%$ ) in the BM, characteristic of human MDS (Figure $1 \mathrm{H}$ ). Then we examined the pathological changes in the femur, liver and spleen. The femur showed extremely active myeloid hyperplasia, mainly in blast cells, with the increased ratio of granulocyte/red cells and abnormal localization of blast precursor cells in RUNX1S291fs mice but not Empty control mice (Figure 1I). Moreover, the RUNX1S291fs mice showed many nucleated cells around the central vein and the portal area of the liver, and exhibited enlargement and irregular shape 
A

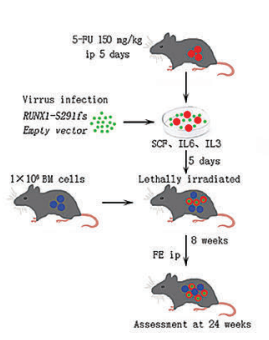

D

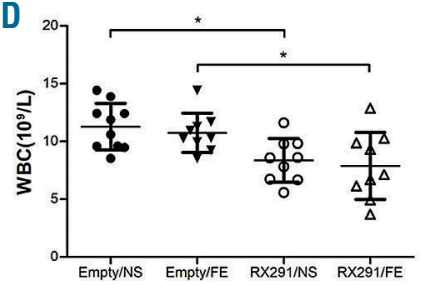

G

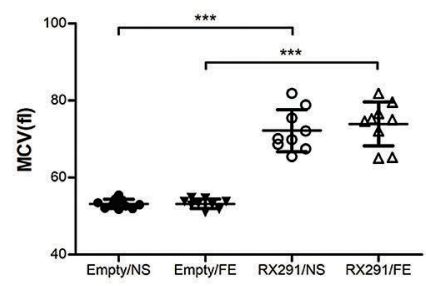

B

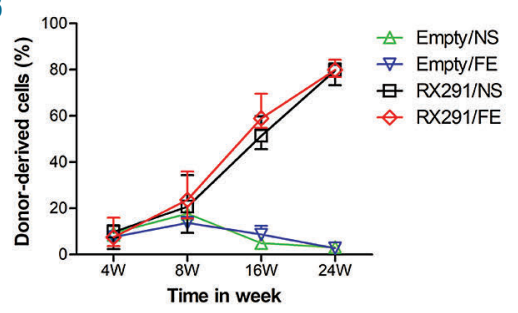

E

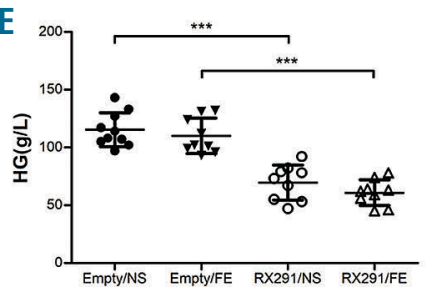

H

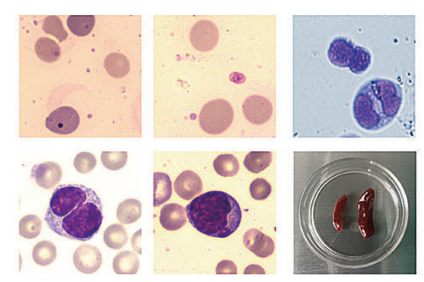

C

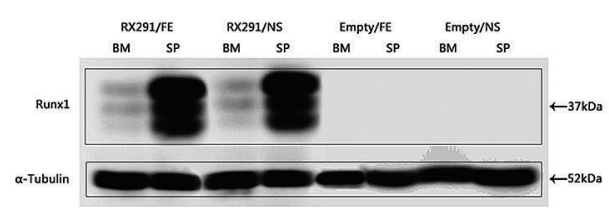

F

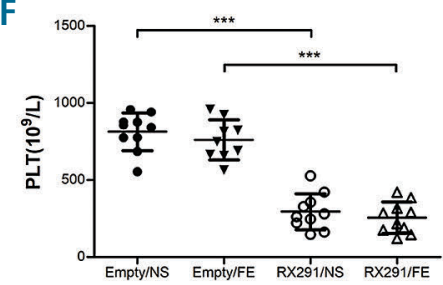

I

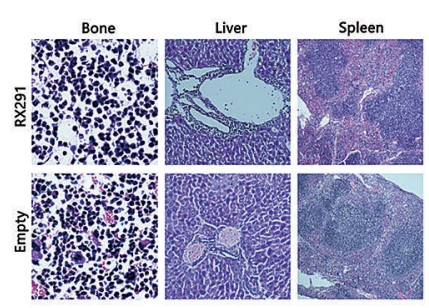

Figure 1. RUNX1S291fs induced mice model can phenotypically recapitulate human myelodysplastic syndrome. (A) Experimental scheme of our model mouse using RUNX1S291fs mutant. (B) Chimerism of GFP+ cells post transplantation. (C) Successful expression of RUNX1S291fs protein detected by western blotting. (D) White blood cell (WBC) count. (E) Hemoglobin (HG) count. (F) Platelet (PLT) count. (G) Mean corpuscular volume (MCV). (H) The morphological abnormality observed in the bone marrow and spleen size. (I) Pathological changes in the femur, liver and spleen. $* P<0.05, * * P<0.01, * * * P<0.001$.

of white pulp and narrow red pulp, whereas such pathological changes were not found in Empty control mice (Figure 1I). Collectively, our RUNX1S291fs-induced mice model showed neutropenia, anemia, thrombocytopenia and multilineage dysplasia, together with less than $20 \%$ blasts, matching the criteria of MDS in the Bethesda proposal and phenotypically recapitulating human MDS.

Next, we examined whether iron overload model can be established in RUNX1S291fs induced MDS mice. We and others previously reported that iron overload can cause liver and spleen enlargement. ${ }^{12,13}$ There was a clear increase in liver and spleen weight in both control mice and in MDS mice administered iron dextran treatment, implying that iron can deposit in the liver and spleen in RUNX1S291fs-induced MDS mice (Figures $1 \mathrm{H}$, and 2A and $B$ ). We further performed Perl's iron staining to verify iron deposition in mice tissues and organs. Significant iron deposition could be observed in the liver, spleen and BM in RX291/FE group compared to RX291/NS mice (Figure 2C), supporting iron overload in RX291/FE mice. In addition, our data showed the level of ferritin was almost undetectable in Empty/NS, while it was significantly higher in RX291/NS mice (Figure 2D), which can be clearly explained by ineffective erythropoiesis in MDS. However, the ferritin in Empty/FE and RX291/FE group was comparable, and was more significant than in Empty/NS and RX291/NS mice, respectively (Figure 2D), indicating that iron overload can also be established in MDS mice. Taken together, administration of iron dextran by intraperitoneal injection confers iron deposition in organs, liver and spleen enlargement, and can be used to facilitate the establishment of an iron overload model in RUNX1S291fsinduced MDS mice.

Iron overload impairs the frequency of normal HSPCS and affects erythroid maturation in MDS mice

To understand how iron overload influences the hematopoietic system, we first evaluated WBC, PLT and HG counts. However, our experiment showed no statistical difference but a downward trend in the PB between the group treated with iron and that treated with normal saline in both control mice and MDS mice (Figure 1D-F). We further analyzed the frequency of HSCs ( $\left.\mathrm{Lin}^{-} \mathrm{c}-\mathrm{Kit}^{+} \mathrm{Sca} 1^{+} / \mathrm{LSK}\right)$ and HPCs ( $\left.\mathrm{Lin}^{-} \mathrm{c}-\mathrm{Kit}^{+} \mathrm{Sca} 1^{-} / \mathrm{LK}\right)$ in BM (Figure $3 \mathrm{~A}$ ) and we found that there were significantly more normal (GFP-) HSCs in MDS mice than in control mice, which may due to extremely active myeloid hyperplasia in MDS. In addition, iron overload can significantly decrease the number of GFP- HSCs in both control and MDS mice. However, we did not observe any significance about mutant (GFP+) HSCs in RX291/NS and RX291/FE mice (Figure $3 \mathrm{~B}$ ). A similar result can be seen regarding the change in normal and mutant HPCs in these groups (Figure 3C). Next, we analyzed erythroid differentiation through CD71 and Ter119 gating (Figure 3D). We referred to proerythroblasts, basophilic erythroblasts, polychro- 
matophilic erythroblasts and orthochromatic erythroblasts as E1, E2, E3 and E4, respectively. Compared to Empty mice, MDS mice showed a significant increase in the number of GFP- E1 and a significant decrease in GFPE2 (Figure 3E), which may due to erythroid dysmaturity in MDS. Of note, iron overload can significantly reduce the frequency of E3 and E4 in control mice and GFP- E1 in MDS mice, while it did not exhibit any significant inhibition in $\mathrm{GFP}^{+} \mathrm{MDS}$ erythroid cells, which may suggest that iron overload has a negative effect on normal hematopoiesis in erythroid, but not malignant clones. Collectively, our study shows that iron overload impairs the frequency of normal HSPCs and may inhibit erythroid maturation in MDS mice.

\section{Iron overload compromises the erythroid} colony-forming capacity of normal HSPCs in MDS mice

We next wanted to examine if iron overload has a negative impact on HSPCs function in MDS mice. Therefore, we collected BM cells and determined the colony-forming capacity or hematopoietic cells reconstitution ability of these cells. Our data showed that normal (GFP-) HSPCs in MDS mice have a significantly lower ability to form colony-forming unit erythroid (CFU-E), burst-forming unit erythroid (BFU-E), and colony-forming unit granulocyte, erythroid, macrophage, megakarycyte (CFU-GEMM) colonies compared to control mice (Figure 4A). What is more, iron overload can markedly inhibit CFU-E and BFU$\mathrm{E}$ of GFP- HSPCs in both control and MDS mice. We also tested a serial replating assay about GFP- HSPCs (Figure $4 \mathrm{~B})$. Despite the fact that the first plating did not show sig- nificantly reduced clonogenic numbers, but there seemed to be a declining trend between Empty/NS and Empty/FE mice, the count of clonogenic HSPCs exhibited a marked decline at the second and subsequent platings (Figure 4B), which supported the view that the colony-forming capacity of normal HSPCs was suppressed by iron overload in MDS mice. Because GFP' HSPCs were unable to form colonies but clusters compared to GFP- HSPCs (Figure 4C), we performed competitive repopulation assay to identify the effect of iron overload on the hematopoietic reconstitution capacity of $\mathrm{GFP}^{+}$cells (Figure 4D). To our surprise, the chimerism of $\mathrm{GFP}^{+}$cells from RX291/FE mice was not significant compared to those of RX291/NS mice (Figure $4 \mathrm{D})$, indicating that iron overload may not damage the hematopoietic reconstitution capacity of $\mathrm{GFP}^{+}$HSPCs. Together, we conclude that iron overload can compromise the erythroid colony-forming capacity of normal HSPCs, but may not affect the hematopoietic reconstitution capacity of abnormal HSPCs in MDS.

Iron overload inhibits erythroid hematopoiesis in MDS mice through ROS

These data showed that iron overload inhibits erythroid hematopoiesis in MDS mice. We next investigated the mechanisms involved in this process. Previous studies have reported that enhanced apoptosis of BM cells in MDS can cause pancytopenia. Indeed, our data showed that the level of apoptosis in RX291/NS and RX291/FE mice was significantly increased compared to Empty/NS and Empty/FE group (Figure 5A), respectively, consistent with previous studies. ${ }^{8}$ In addition, iron overload can
A

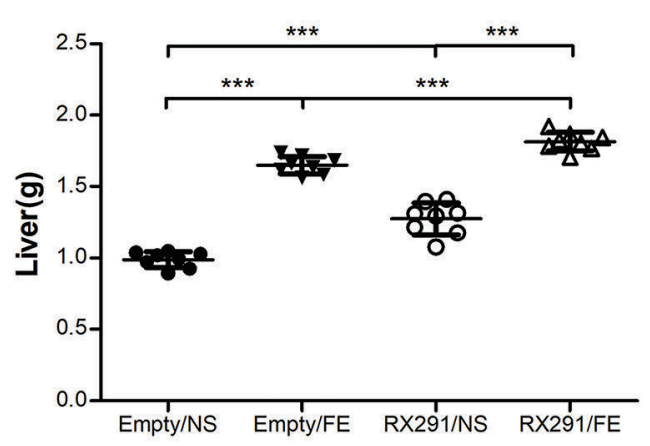

C

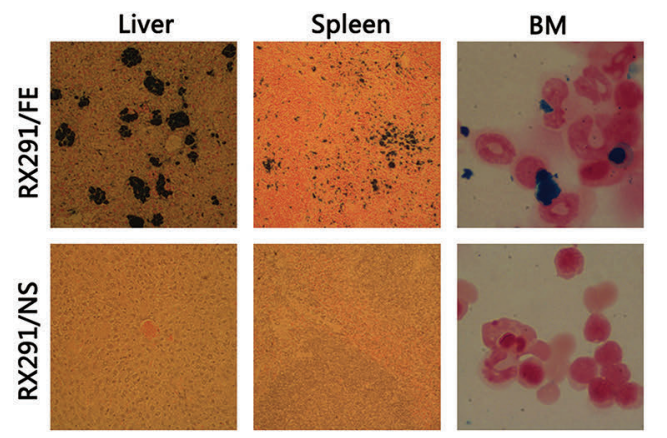

B

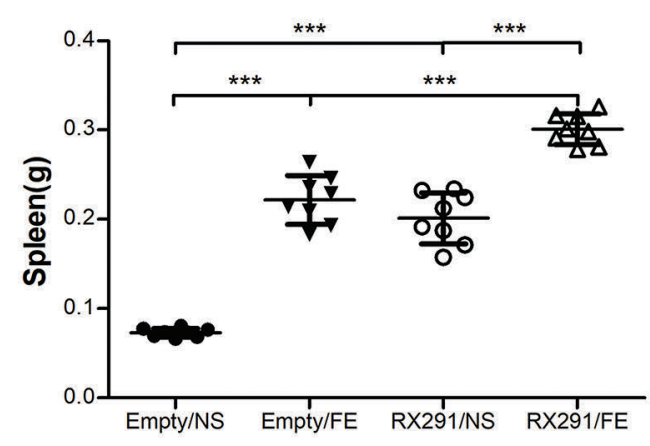

D

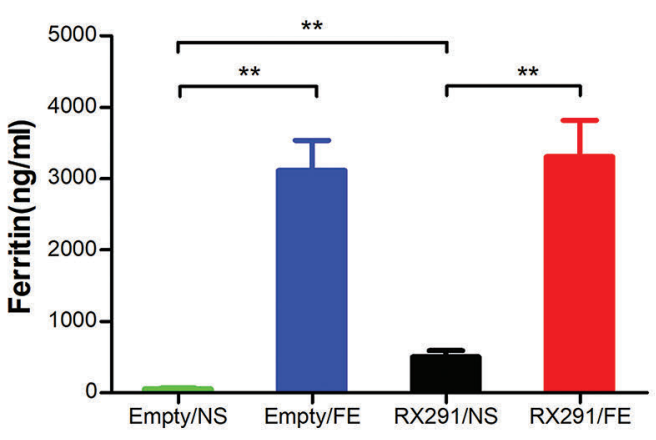

Figure 2. Iron overload model can be established in RUNX1S291fs induced myelodysplastic syndrome mice. (A) Weight of liver. (B) Weight of spleen. (C) Perl's iron staining of liver, spleen and bone marrow (BM). (D) Ferritin level detected by ELISA assay. ${ }^{*} P<0.01, * * * P<0.001$. 
induce apoptosis of BM cells in normal mice (Figure 5A), which has previously been confirmed by others. ${ }^{14}$ Of note, normal (GFP-) BM cells in RX291/FE mice have a markedly higher apoptosis level than those of RX291/NS mice, but not mutant $\left(\mathrm{GFP}^{+}\right)$cells (Figure 5A), implying that iron overload can promote apoptosis of normal BM cells in MDS. It has been reported that ROS can induce apoptosis. ${ }^{14}$ Our data showed that the ROS level was also clearly higher in RX291/FE mice than that of RX291/NS mice (Figure 5B) in BM. And erythroid cells in BM presented similar changes (Figure $5 \mathrm{C}$ ). We also detected the mRNA levels of NOX4 related to ROS gen-
A

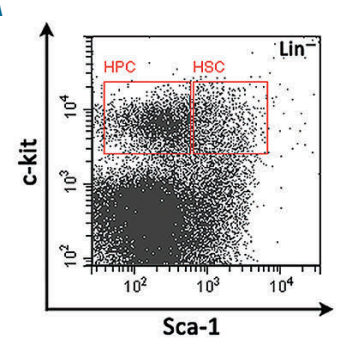

D

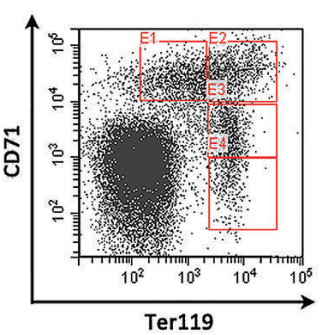

B

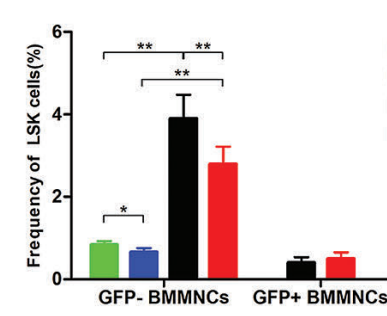

E

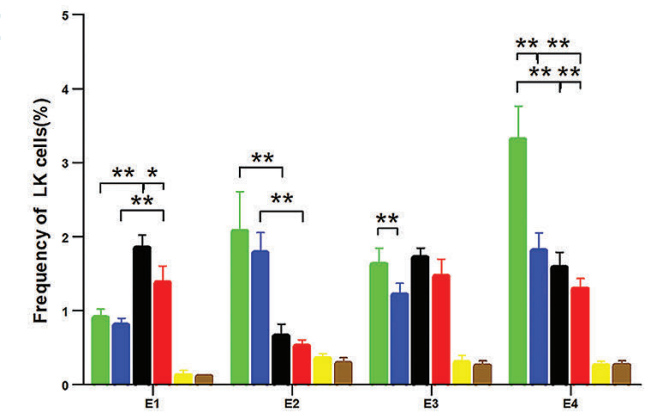

C

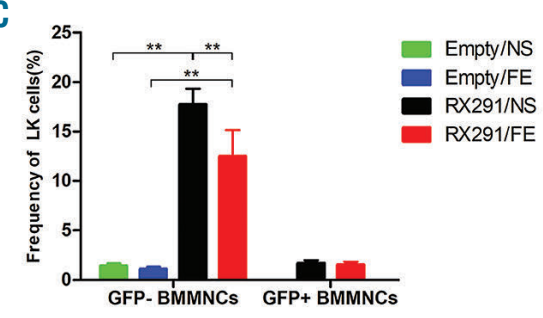

Figure 3. Iron overload impairs the frequency of normal hematopoietic stem and progenitor cells (HSPCs) and affects erythroid maturation in myelodysplastic syndrome (MDS) mice. (A) Gating hematopoietic stem cells (HSCs) and hematopoietic progenitor cells (HPCs) by flow cytometry. (B) The frequency of HSCs in different groups. (C) Frequency of HPCs in different groups. (D) Different stages of erythroid through CD71 and Ter119 gating. (E) Effect of iron overload on erythroid differ-

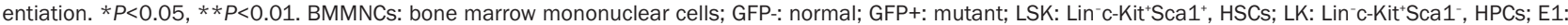
proerythroblasts; E2: basophilic erythroblasts; E3: polychromatophilic erythroblasts; E4: orthochromatic erythroblasts.

A

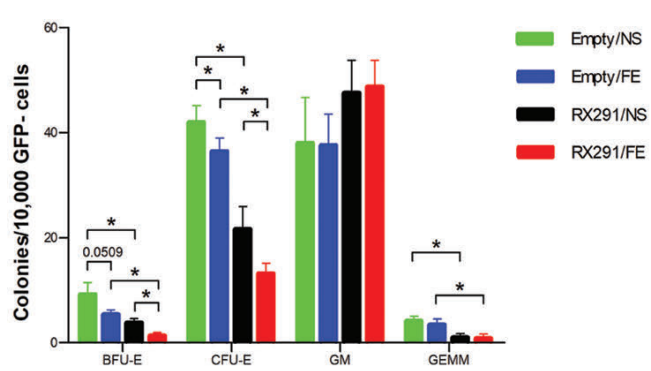

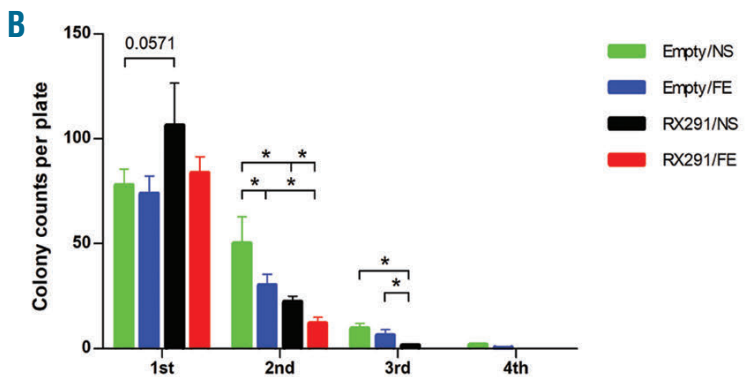

D
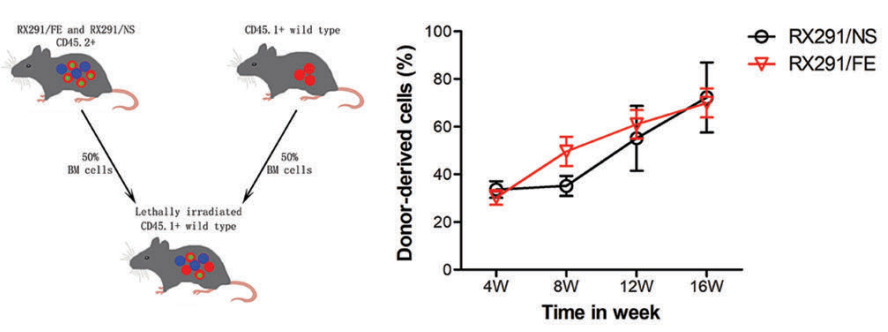

Figure 4. Iron overload compromises the erythroid colony-forming capacity of normal hematopoietic stem and progenitor cells (HSPCs) in myelodysplastic syndrome mice. (A) The colonies generated by GFP- cells. (B) A serial replating assay about GFP- HSPCs. (C) Comparison of the colony-forming capacity between GFPand $\mathrm{GFP}^{+}$cells. Left column is in bright field; right column is in fluorescence field. Red arrow indicates the location of GFP cells. (D) Experimental scheme of our mouse model using $\mathrm{GFP}^{+}$cells to perform competitive repopulation assay and detecting chimerism of GFP ${ }^{+}$cells post transplantation. ${ }^{*} P<0.05$. CFU-E: colony-forming unit erythroid; BFU-E: burst-forming unit erythroid; GM: colony-forming unit granulocyte-macrophage; GEMM: colony-forming unit granulocyte, erythroid, macrophage, megakarycyte. 
eration and GPX1 involved in ROS clearance in erythroid cells. Interestingly, the expression of NOX4 was significantly higher in RX291/FE mice than that of RX291/NS mice (Figure 5D), while the level of GPX1 was significantly lower in the RX291/FE group (Figure 5E), supporting the view that iron overload can induce ROS to damage erythroid hematopoiesis. Previous studies have reported that TGF- $\beta$ signaling is myelosuppressive and inhibits erythroid differentiation by induction of ROS and apoptosis in erythroblasts. ${ }^{15-17}$ We then examined the mRNA levels of TGF- $\beta$ superfamily including GDF8, GDF11, GDF15, Activin A, Activin B, Acvr2b, ALK4, and $A L K 5$, and found that GDF11 mRNA levels were significantly increased in RX291/FE mice compared to RX291/NS mice (Figure 5F). We further detected the GDF11 protein levels in serum, which showed similar results in these two groups (Figure 5G). Collectively, iron overload can damage erythroid hematopoiesis in MDS mice, which may partially be due to GDF11-induced ROS, leading to enhanced apoptosis of normal BM cells and inhibition of their function in MDS.

\section{Iron overload shortens survival MDS mice}

Given that iron overload results in a suppressive effect in the frequency and function of normal HSPCs in MDS mice, we postulated that iron overload can affect the median survival of these mice. The mice were observed for 360 days post transplantation and each group has more than ten mice. In our study, MDS mice have an obviously shorter median survival time than control mice (Figure 6), mostly due to AML transformation. For control mice, the survival time did not differ between Empty/NS and Empty/FE mice because the experiment was terminated at the end of day 360. However, RX291/FE mice exhibited a significantly shorter median survival than RX291/NS mice (Figure 6), suggesting that iron overload can shorten the survival time of MDS mice.

\section{Discussion}

Myelodysplastic syndrome is a heterogeneous group of clonal stem cell disorders characterized by multilineage dysplasia, ineffective hematopoiesis, pancytopenia, and with a high risk of developing into acute myeloid leukemia. ${ }^{18}$ Over recent years, investigators all over the world have been trying hard to establish an MDS model to further study this disease. However, only a few mouse models are available for human MDS. RUNX1 is a frequently mutated gene in MDS7 and RUNX1S291fs is one of the most common mutations in RUNX1. It has been demonstrated that transduction of this mutant into BM cells can be used to construct MDS mice. ${ }^{8}$ Indeed, our RUNX1S291fs-induced mice showed neutropenia, anemia, thrombocytopenia, multilineage dysplasia, together with less than $20 \%$ blasts, matching the criteria of MDS in the Bethesda proposal and phenotypically recapitulating human MDS.

Due to frequent blood transfusions and ineffective hematopoiesis, most MDS patients will eventually develop iron overload. Excessive iron deposits in the tissues and organs cause a series of complications, affecting the prognosis of MDS patients. In turn, iron overload has a negative role in hematopoiesis in MDS, further deteriorating the situation of these patients. To understand how iron overload influences hematopoiesis in MDS, we first established an iron overload model in RUNX1S291fsinduced MDS mice by intraperitoneal injection of iron dextran. Our data presented more iron deposition in organs, and more significant liver and spleen enlargement in RX291/FE mice, supporting the successful establishment of an iron overload model in RUNX1S291fs-induced MDS mice. In the present study, although there was no statistical significance in the levels of WBC, PLT and HG in the PB between Empty/NS and Empty/FE, we observed a declining trend in Empty/FE relative to Empty/NS mice,
A

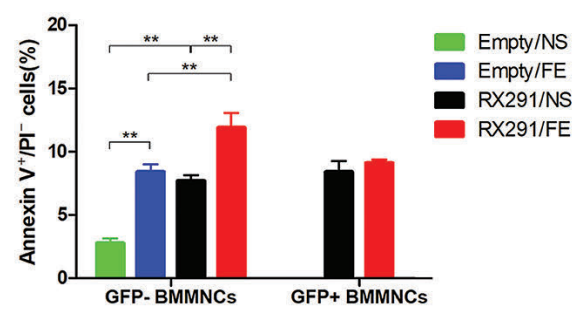

D

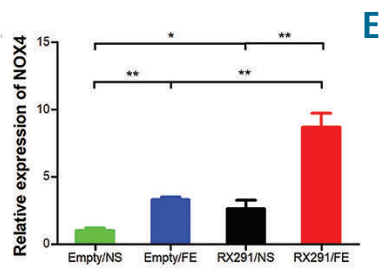

$\mathrm{E}$

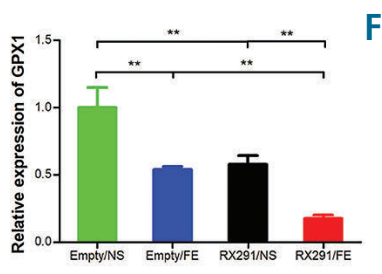

B

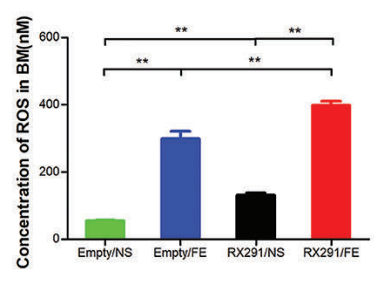

$F$

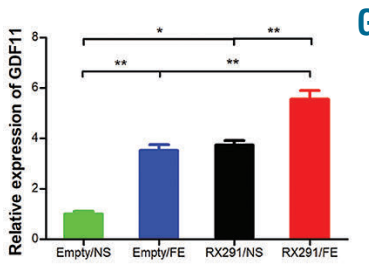

C

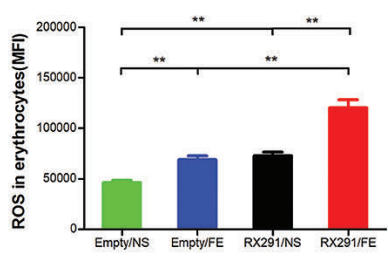

G

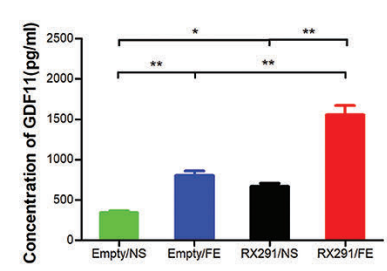

Figure 5. Iron overload inhibits erythroid hematopoiesis in myelodysplastic syndrome (MDS) mice through reactive oxygen species (ROS). (A) Level of apoptosis in MDS mice. (B) ROS level in the bone marrow (BM) and erythrocytes. (C) ROS level in the erythrocytes. (D) mRNA expression of NADPH oxidase 4 (NOX4) gene. (E) mRNA expression of glutathione peroxidase 1 (GPX1) gene. (F) mRNA expression of growth differentiation factor 11 (GDF11) gene. (G) The concentration of GDF11 protein in serum detected by ELISA assay. $* P<0.05, * * P<0.01, * * * P<0.001$. BMMNC: bone marrow mononuclear cells. 


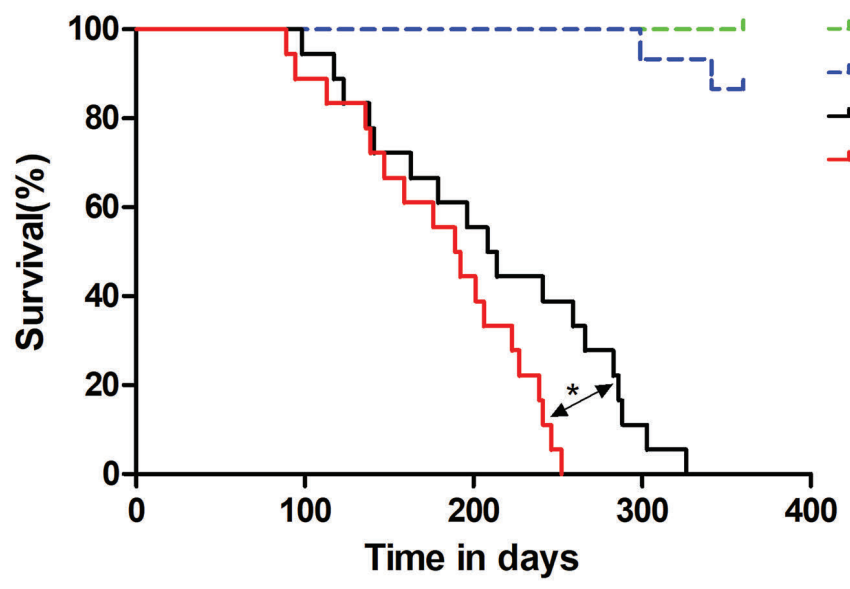

-L. Empty/NS

-ᄂ. Empty/FE

- RX291/NS

$+\mathrm{RX} 291 / \mathrm{FE}$
Figure 6. Iron overload shortens the survival time of myelodysplastic syndrome mice. similar to our previous study. ${ }^{13}$ We also obtained similar results in the $\mathrm{PB}$ between the two groups of MDS mice. In addition, we found that iron overload impairs the frequency of normal HSPCs, especially in erythroid cells, but not abnormal HSPCs in MDS mice. We further investigated the impact of iron overload on HSPCs function and found that iron overload compromises the erythroid colonyforming capacity of normal HSPCs, but may not affect the hematopoietic reconstitution capacity of abnormal HSPCs in MDS mice. In addition, RX291/FE mice have a shorter median survival than RX291/NS mice, indicating that iron overload can shorten survival time in MDS mice. There are increasing data to show that oxidative stress was increased in BM cells of patients with iron overload, and antioxidant or iron chelator therapy could partially rescue the impaired hematopoietic function of patients, which indicates the presence of ROS-induced cellular injury. ${ }^{19,23}$ Of interest, we detected the level of ROS and found it significantly increased in RX291/FE compared with RX291/NS group, suggesting that iron overload impairs normal HSPCs, at least in part, via inducing ROS in MDS, consistent with previous studies. ${ }^{19,24}$ Our data showed that iron overload decreased the number of HSPCs partially due to ROS-induced apoptosis. However, whether the reduced HSPCs was regulated by HIF-1a/ROS or NF-кB pathway warrants further investigation. ${ }^{20,24}$ It has been reported that the TGF- $\beta$ pathway is myelosuppressive and inhibits erythroid differentiation by induction of ROS and apoptosis in erythroblasts. ${ }^{15-17,25}$ Our data showed in GDF11, one of the TGF- $\beta$ superfamilies, mRNA and protein levels significantly increased in RX291/FE mice compared to RX291/NS mice, suggesting that iron overload can damage erythroid hematopoiesis in MDS mice, which may partially be due to GDF11-induced ROS, leading to enhanced apoptosis of normal BM cells and inhibition of their function in MDS. However, additional studies are needed to clarify whether GDF11-induced ROS and apoptosis of erythroid was related to the Fas-Fas ligand path- way. ${ }^{16,25}$ Interestingly, Masayo et al. found that iron overload can activate glucose metabolism and increase DNA methylation, which is associated with MDS pathogenesis and progression, and iron chelation can reverse these effects. ${ }^{26}$ Therefore, future studies should be conducted to evaluate the impact of iron overload on metabolic pathways such as glucose and lipid involved in MDS pathogenesis in our model. In addition, our mice can be used to illustrate the association between oxidative imbalance and iron overload in MDS. ${ }^{18}$ Our previous study showed that damaged mesenchymal stromal cells (MSC) were related to iron overload induced ROS in normal mice. ${ }^{21}$ However, Zheng et al. reported that iron overload damages MSC through AMPK/MFF/Drp1 pathway in MDS. ${ }^{27}$ Thus, further research should investigate the effect of iron overload on the BM microenvironment in MDS.

In conclusion, our preliminary findings suggest that iron overload impairs the frequency and function of normal HSPCs, particularly in erythroid, at least in part via GDF11-induced ROS, and shortens survival in MDS. Given that there are a few MDS models available, and we are the first to utilize RUNX1-S291fs-induced MDS mice to successfully construct an iron overload model, we hope this model will be helpful for further exploring the influence and mechanism of iron overload on MDS.

\section{Acknowledgments}

The authors would like to thank Dr Atsushi Iwam for the valuable plasmids. We also thank Dr Gang Huang for excellent technical assistance.

\section{Funding}

This work was supported by grants from the National Natural Sciences Foundation of China (81400092), Tianjin Key Natural Science Foundation (17JCZDJC35800, 15JCQNJC45500), and Tianjin Key Science and Technology Program (2015K215, 15KG134, 16KG110), as well as Tianjin First Central Hospital. 


\section{References}

1. Harada H. MDS: Recent progress in molecular pathogenesis and clinical aspects. Rinsho Ketsueki. 2017;58(10):1941-1950.

2. Ganguly BB, Banerjee D, Agarwal MB. Impact of chromosome alterations, genetic mutations and clonal hematopoiesis of indeterminate potential (CHIP) on the classification and risk stratification of MDS. Blood Cells Mol Dis. 2018;69:90-100.

3. Imran F, Phatak P. Decision points in the treatment of transfusional iron overload in patients with myelodysplastic syndromes: why, when, and how to chelate. Expert Rev Hematol. 2017;10(1):53-64.

4. Zeidan AM, Pullarkat VA, Komrokji RS. Overcoming barriers to treating iron overload in patients with lower-risk myelodysplastic syndrome. Crit Rev Oncol Hematol. 2017;117:57-66.

5. Gattermann N. Iron overload in myelodysplastic syndromes (MDS). Int J Hematol. 2018;107(1):55-63

6. Wong C, Wong S, Leitch HA. Iron overload in lower international prognostic scoring system risk patients with myelodysplastic syndrome receiving red blood cell transfusions: Relation to infections and possible benefit of iron chelation therapy. Leuk Res. 2018;67:75-81.

7. Sood R, Kamikubo Y, Liu P. Role of RUNX1 in hematological malignancies. Blood. 2017;129(15):2070-2082.

8. Sashida G, Harada H, Matsui H, et al. Ezh2 loss promotes development of myelodysplastic syndrome but attenuates its predisposition to leukaemic transformation. Nat Commun. 2014;5:4177.

9. Morita S, Kojima T, Kitamura T. Plat-E: an efficient and stable system for transient packaging of retroviruses. Gene Ther. 2000;7(12):1063-1066.

10. Unnisa Z, Clark JP, Roychoudhury J, et al.
Meis1 preserves hematopoietic stem cells in mice by limiting oxidative stress. Blood. 2012;120(25):4973-4981.

11. Kogan SC, Ward JM, Anver MR, et al. Bethesda proposals for classification of nonlymphoid hematopoietic neoplasms in mice. Blood. 2002;100(1):238-245.

12. Ramanathan G, Olynyk JK, Ferrari P. Diagnosing and preventing iron overload. Hemodial Int. 2017;21 Suppl 1:S58-S67.

13. Chai X, Li D, Cao X, et al. ROS-mediated iron overload injures the hematopoiesis of bone marrow by damaging hematopoietic stem/progenitor cells in mice. Sci Rep. 2015;5:10181.

14. Muto Y, Nishiyama M, Nita A, Moroishi I Nakayama KI. Essential role of FBXL5mediated cellular iron homeostasis in maintenance of hematopoietic stem cells. Nat Commun. 2017;8:16114.

15. Mies A, Platzbecker U. Increasing the effectiveness of hematopoiesis in myelodysplastic syndromes: erythropoiesis-stimulating agents and transforming growth factor-beta superfamily inhibitors. Semin Hematol. 2017;54(3):141-146.

16. Dussiot M, Maciel TT, Fricot A, et al. An activin receptor IIA ligand trap corrects ineffective erythropoiesis in beta-thalassemia. Nat Med. 2014;20(4):398-407.

17. Kim A, Nemeth E. New insights into iron regulation and erythropoiesis. Curr Opin Hematol. 2015;22(3):199-205.

18. Ivars $\mathrm{D}$, Orero $M T$, Javier $\mathrm{K}$, et al. Oxidative imbalance in low/intermediate1-risk myelodysplastic syndrome patients: The influence of iron overload Clin Biochem. 2017;50(16-17):911-917.

19. Angelucci E, Cianciulli P, Finelli C, Mecucci C, Voso MT, Tura S. Unraveling the mechanisms behind iron overload and ineffective hematopoiesis in myelodysplastic syndromes. Leuk Res. 2017;62:108-115.

20. Meunier M, Ancelet S, Lefebvre C, et al.
Reactive oxygen species levels control NFkappaB activation by low dose deferasirox in erythroid progenitors of low risk myelodysplastic syndromes. Oncotarget. 2017;8(62):105510-105524.

21. Zhang Y, Zhai W, Zhao M, et al. Effects of iron overload on the bone marrow microenvironment in mice. Plos One. 2015;10(3):e120219.

22. Lu W, Zhao M, Rajbhandary S, et al. Free iron catalyzes oxidative damage to hematopoietic cells/mesenchymal stem cells in vitro and suppresses hematopoiesis in iron overload patients. Eur J Haematol. 2013;91(3):249-261.

23. Chen J, Lu WY, Zhao MF, et al. Reactive oxygen species mediated $\mathrm{T}$ lymphocyte abnormalities in an iron-overloaded mouse model and iron-overloaded patients with myelodysplastic syndromes. Ann Hematol. 2017;96(7):1085-1095.

24. Zheng QO, Zhao YS, Guo J, et al. Iron overload promotes erythroid apoptosis through regulating HIF-1a/ROS signaling pathway in patients with myelodysplastic syndrome. Leuk Res. 2017:58:55-62.

25. Arlet JB, Dussiot M, Moura IC, Hermine O, Courtois G. Novel players in beta-thalassemia dyserythropoiesis and new therapeutic strategies. Curr Opin Hematol. 2016;23(3):181-188.

26. Yamamoto $M$, Tanaka $H$, Toki $Y$, et al Iron-induced epigenetic abnormalities of mouse bone marrow through aberrant activation of aconitase and isocitrate dehydrogenase. Int J Hematol. 2016;104(4):491501.

27. Zheng $\mathrm{Q}$, Zhao Y, Guo J, et al. Iron overload promotes mitochondrial fragmentation in mesenchymal stromal cells from myelodysplastic syndrome patients through activation of the AMPK/MFF/Drp1 pathway. Cell Death Dis. 2018;9(5):515 